\title{
A pilot study of essential tremor: cerebellar GABA+/Glx ratio is correlated with tremor severity
}

\author{
Sofie Tapper ${ }^{1,2+}$, Nathanael Göransson ${ }^{3 \dagger}$, Peter Lundberg ${ }^{1,2}$, Anders Tisell ${ }^{1,2^{*}}$ (D) and Peter Zsigmond ${ }^{4}$
}

\begin{abstract}
Objective: Essential tremor is a common movement disorder with an unclear origin. Emerging evidence suggests the role of the cerebellum and the thalamus in tremor pathophysiology. We examined the two main neurotransmitters acting inhibitory (GABA+) and excitatory (GIX) respectively, in the thalamus and cerebellum, in patients diagnosed with severe essential tremor. Furthermore, we also investigated the relationship between determined neurotransmitter concentrations and tremor severity in the essential tremor patients.

Methods: Ten essential tremor patients (prior to deep brain stimulation surgery) and six healthy controls, were scanned using a 3T MR system. GABA+ and Glx concentrations were measured using magnetic resonance spectroscopy (MRS) performed using single voxel MEGA-PRESS. For the purpose of assessing the tremor severity, the essential tremor rating scale (ETRS) was used in accordance with Fahn, Tolosa, and Marin.

Results: We demonstrated that the cerebellar GABA+/Glx ratio was positively correlated to the ETRS $(r=0.70, p=$ 0.03 in essential tremor. Cerebellar and thalamic GABA+ and Glx concentrations did not show any significant difference when comparing essential tremor patients with healthy controls, at the group level.

Conclusion: We demonstrated a positive correlation between increasing tremor disability and the ratio of GABA+/ Glx in the cerebellum of essential tremor patients. This highlights the impact of an altered balance of the excitatory and inhibitory neurotransmitters in tremor severity. Rather than a change in GABA+, which was constant, we attribute this finding to an overall decrease of Glx.
\end{abstract}

Keywords: Essential tremor, GABA, Glutamate, Magnetic resonance spectroscopy, MEGA-PRESS

\section{Introduction}

Essential tremor (ET) is one of the most common movement disorders with a prevalence of $4.6 \%$ in patients over 65 years old [1]. The diagnosis of ET is generally based on a thorough analysis of clinical history, including a neurological examination by a movement disorder

\footnotetext{
* Correspondence: Anders.Tisell@liu.se

†Sofie Tapper and Nathanael Göransson contributed equally to this work. ${ }^{1}$ Center for Medical Image Science and Visualization (CMIV), Linköping University, Linköping, Sweden

${ }^{2}$ Department of Medical Radiation Physics and Department of Health, Medicine and Caring Sciences, Linköping University, Linköping, Sweden Full list of author information is available at the end of the article
}

specialist. The upper limbs are affected in $95 \%$ of patients, that result in shaking of the hands and forearms during simple voluntary movements [2,3]. In addition, a wide range of other symptoms can appear (cognitive, psychiatric and other motor symptoms); the clinical diversity makes ET a very heterogenic disease [3, 4]. Not surprisingly, the pathophysiology of ET is therefore still not completely understood [5].

Recent advances in neuroimaging and spectroscopy opens up for the study of a novel range of structural, functional and metabolic changes in these patients [5]. Resting state functional MRI (fMRI) studies have shown 
decreased functional connectivity of the cerebellar network in ET [6]. Also significant alterations have been observed in the cerebello-thalamo-cortical network in patients with ET, which correlated with the overall tremor severity [7]. In addition, this network is the most common target for deep brain stimulation (DBS) surgery in ET. Several imaging studies support a cerebellar involvement in ET, where for example alterations in the grey matter volume of several brain regions including the cerebellum in ET has been observed [8]. An fMRI study showed an association between tremor and ipsilateral cerebellar activity [9], and a 'positron emission tomography' (PET) study showed an increased cerebellar blood flow in ET [10]. These and other studies suggests that both the cerebellum and the thalamus play a central role in the pathophysiology of ET [11-13].

Postmortem studies have shown structural changes in the cerebellum of patients with ET [14-16], including a reduced number of 'Purkinje cells' (PCs), dendritic swelling and increased Purkinje cell torpedoes in the cerebellum [14] (i.e., neurodegeneration). PCs propagate an input to the deep cerebellar nuclei, dentate nucleus, through gamma-aminobutyric acid (GABA) which is an inhibitory neurotransmitter. A postmortem study showed a decreased number of GABA receptors in the dentate nucleus, $\mathrm{GABA}_{\mathrm{A}}$ (35\% reduction) and $\mathrm{GABA}_{\mathrm{B}}$ (ca. 25\% reduction) [17]. Moreover, PET studies using Flumazenil, which specifically binds to the $\mathrm{GABA}_{\mathrm{A}}$ receptor have shown an increased binding in the cerebellum, but also in the thalamus and the premotor cortex [18]. Moreover, the severity of tremor has been correlated to abnormalities found in the GABA receptor binding [19], and several GABA-ergic drugs have been shown to relieve ET [20].

Climbing fibers (CF) of the cerebellum use the excitatory neurotransmitter glutamate (Glu) (see Fig. 4), and anomalies of CF-PC synaptic connections with decreased climbing fiber synaptic density in the cerebellar cortex have also been observed [15]. An altered distribution of climbing fibers and synapses extending into the outer $20 \%$ of the molecular layer, 'Parallel fiber' (PF) territory, has been reported $[15,16]$, and it has been shown that tremor severity correlates inversely with synapse CF-PC abnormalities in the PF area [21].

'Proton magnetic resonance spectroscopy' $\left({ }^{1} \mathrm{H}\right.$-MRS) is a tool for non-invasive metabolite quantification, including $N$-acetyl-L-aspartate (NAA), total choline (tCho), and total creatine $(\mathrm{tCr})$. It is therefore a powerful tool to quantitatively investigate the biochemistry and metabolism in the human brain in relation to both pathologies and healthy conditions. Greater magnetic field strength (such as $3 \mathrm{~T}$ instead of $1.5 \mathrm{~T}$ ) allows for an increased signal-to-noise-ratio, better temporal resolution, and also provides a larger spectral dispersion which significantly improves quantification [22]. The latter allows for the detection of metabolites of lower concentrations than otherwise.

Louis et al. investigated the GABA concentrations in large voxels including the dentate nucleus by the use of MRS. Interestingly they found that the right dentate nucleus GABA concentration was significantly higher than the GABA concentration obtained from the left side, only observed for the ET patients [23].

There seems to be a consensus about the involvement of the cerebellum and thalamus in ET, although the potential neurochemical abnormalities causing ET remain to be further elucidated. Our aim in this study was therefore to evaluate the relationship between glutamate (Glu, determined as Glx) and GABA (determined as GABA+) concentrations in the cerebellum and thalamus, in patients diagnosed with severe ET immediately prior to DBS intervention, and to compare the concentrations of these with those obtained by examining a healthy control group. Furthermore, we aimed to investigate the correlation between neurotransmitter concentrations in the ET group and the tremor severity as determined using an ET rating scale (ETRS) [24], in order to facilitate further conclusions with respect to the underlying pathology, relationship to debilitating tremor severity and metabolic alterations.

\section{Materials and methods}

This study was approved by the local ethics committee at the University Hospital in Linköping (Ref. No. 2013/ 403-31, P. Zsigmond), and informed written consent was received from the patients.

\section{Subject description}

Ten right-handed Caucasian ET patients $(60.2 \pm 9.7$ years, seven males, Table 1), and six right-handed Caucasian healthy controls (HCs) $(62.2 \pm 11.4$ years, five males) were included in this study. The patients were referred to the neurosurgical department in Linköping after being assessed and diagnosed by a movement disorder specialist. All anti tremor medication was withdrawn one week prior to MRexamination.

To assess the tremor severity in the patients and controls, the 'essential tremor rating scale' (ETRS) was used in accordance with Fahn, Tolosa, and Marin [24]. Only the dominant extremity was evaluated since this is the clinical routine in the department. Thus, the right upper extremity was evaluated with part A item 5 and part B items 10-14. The ETRS score was defined as the total sum score of these evaluated items, and the maximal score was 32 points (Supplementary Table 1). 
Table 1 Description of the ET patients. Each patent's age and sex, history of ET, if treated with medication, if a current smoker, and the weekly ethanol intake are summarized. The ETRS score, thus the sum of the evaluated items, is also shown for each patient

\begin{tabular}{|c|c|c|c|c|c|c|c|}
\hline Patient & Age & Sex & History of ET & Medication & Smoker & Ethanol & ETRS score \\
\hline 1 & 51 & M & $>20$ years & 0 & No & $<1$ drink/week & 21 \\
\hline 2 & 76 & M & $>10$ years & 0 & No & $<1$ drink/week & 14 \\
\hline 3 & 67 & M & $>10$ years & 0 & No & $<1$ drink/week & 23 \\
\hline 4 & 67 & M & $>10$ years & 0 & No & $<1$ drink/week & 16 \\
\hline 5 & 41 & M & 10 years & 0 & No & $<1$ drink/week & 15 \\
\hline 6 & 59 & $\mathrm{~F}$ & 8 years & 0 & Yes & 1 drink/day & 21 \\
\hline 7 & 69 & $\mathrm{~F}$ & 4 years & 0 & No & $<1$ drink/week & 17 \\
\hline 8 & 60 & M & $>15$ years & 0 & No & $<1$ drink/week & 17 \\
\hline 9 & 63 & $\mathrm{~F}$ & $>20$ years & 0 & No & $<1$ drink/week & 15 \\
\hline 10 & 53 & M & $>20$ years & 0 & No & $<1$ drink/week & 17 \\
\hline
\end{tabular}

\section{Magnetic resonance measurements}

The ET patients and HCs were scanned using a $3 \mathrm{~T}$ Philips Ingenia MR system (Philips Healthcare, Best, Netherlands), equipped with a 12-channel phased array head coil. To assure correct voxel placements in the MRS measurements, $\mathrm{T}_{2}$-weighted turbo spin echo images were acquired in coronal (Res $=0.5 * 0.5 * 4 \mathrm{~mm}^{3}$, FOV $=230 * 230 * 96)$, sagittal (Res $=0.5 * 0.5 * 4 \mathrm{~mm}^{3}$, FOV $=$ $242 * 242 * 96 \mathrm{~mm}^{3}$ ), and axial (Res: $0.5 * 0.5 * 2 \mathrm{~mm}^{3}$ FOV = $250 * 250 * 140 \mathrm{~mm}^{3}$ ) orientations. Furthermore, the MRS measurements were carried out using a single voxel MEGA-PRESS pulse sequence [25-27] optimized for GABA quantification $(\mathrm{TR} / \mathrm{TE}=2000 / 68 \mathrm{~ms}$, editing pulse $\mathrm{ON}$ at $1.90 \mathrm{ppm}$, editing pulse OFF at $7.46 \mathrm{ppm}$, water suppression MOIST, eight phase cycle steps, 20 OFF and $20 \mathrm{ON}$ dynamics, total acquisition time of 10 min and $40 \mathrm{~s}$ ). A total of four MEGA-PRESS measurements were performed, two with the voxel $(30 \times 30 \times 30$ $\mathrm{mm}^{3}$, Fig. 1a) placed bilaterally in the thalamic region, and two with the voxel $\left(35 \times 25 \times 25 \mathrm{~mm}^{3}\right.$, Fig. 1d)

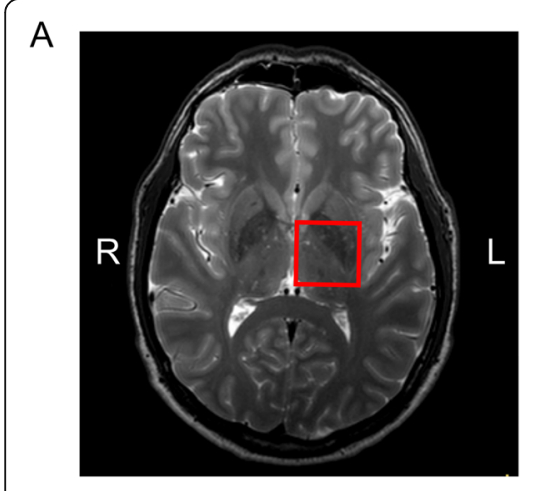

D

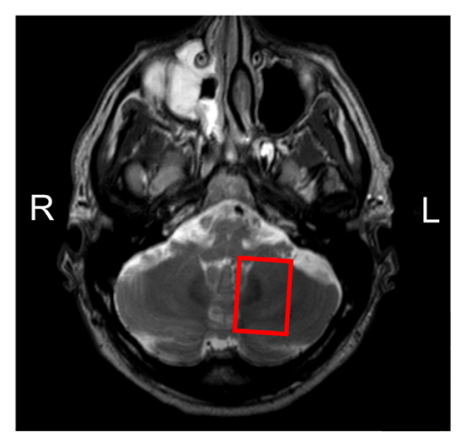

\section{B}

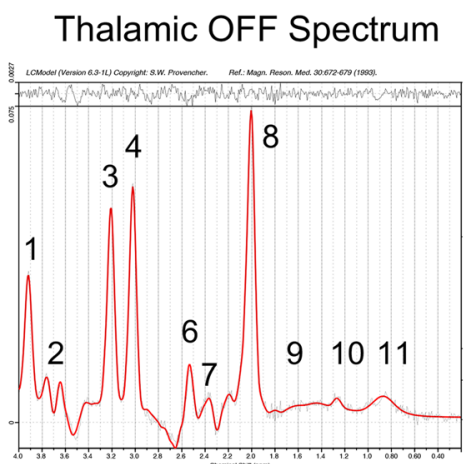

E

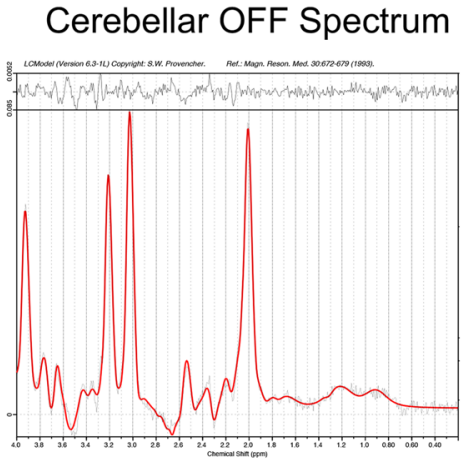

\section{Thalamic GABA+ Spectrum}

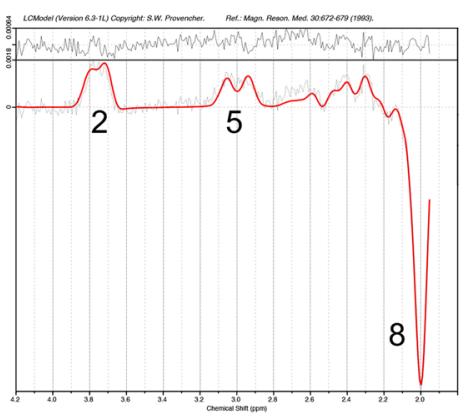

$\mathrm{F}$

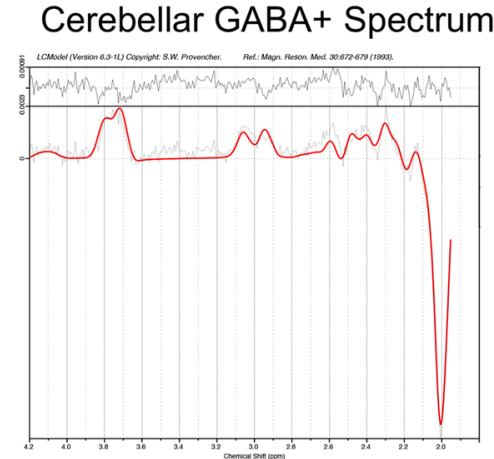

Fig. 1 Representative voxel placements. Representative voxel placements (a and $\mathbf{d})$ and corresponding thalamic (b-c) and cerebellar (e-f) spectra. Assignments: 1, Creatine $\left(-{ }^{2} \mathrm{CH}_{2}-\right) ; 2$, Glx $\left(-{ }^{2} \mathrm{CH}-\right) ; 3$, Choline $\left(-\mathrm{N}\left(\mathrm{CH}_{3}\right)_{3}\right) ; 4$, Creatine $\left(-\mathrm{N}\left(\mathrm{CH}_{3}\right)\right) ; 5, \mathrm{GABA}+\left(-{ }^{4} \mathrm{CH}_{2}-\right) ; 6$, tNA $\left(-{ }^{3} \mathrm{CH}_{2}-\right) ; 7, \mathrm{Glx}\left(-{ }^{4} \mathrm{CH}_{2}-\right)$; 8, tNA $\left(-{ }^{2} \mathrm{CH}_{3}\right) ; 9-11$, Macromolecules and lipids, $-\mathrm{CH}_{2}$ - Abbreviations: Glx, glutamate+glutamine; GABA+, $\mathrm{y}$-Aminobutyric acid (+macromolecule signal); tNA, total N-acetylaspartate (NAA + NAAG) 
placed bilaterally in the cerebellar region. The placement of the voxel in the anatomy was performed by two trained neurosurgeons ensuring a comparable placement among the ET cases and healthy controls. The reproducibility of the MEGA-PRESS measurements in cerebellar region was investigated and reported elsewhere [28]. After each MEGA-PRESS measurement, a shorter identical measurement but with unsuppressed water, was acquired to obtain a reference of water within the tissue in the voxel. This measurement was used for phase correction and water scaling of the resulting concentrations. Finally, since no macromolecular suppression was performed when acquiring the data in this study, the term GABA+ (GABA plus co-edited macromolecular signals) will be used throughout this report.

The MRS datasets were phase-corrected according to Klose [29] and frequency-aligned prior to averaging. For the purpose of GABA+ quantification, a resulting difference spectrum was computed by subtracting the averaged OFF spectrum from the averaged ON spectrum. Furthermore, due to the difficulty in separating glutamate from glutamine in the spectra, these concentrations are often reported as a combined measurement termed 'Glx'. Only OFF data, which were unaffected by the GABA-editing pulses were used in the Glx quantification. After post-processing of the data, the difference spectra and averaged OFF spectra were quantified using LCModel (Version 6.3-1 L) [30], using simulated basis sets obtained from the Dydak Lab (December 14th 2018) [31, 32]. Figure 1 shows representative thalamic (B-C) and cerebellar (E-F) spectra fitted by LCModel. Finally, as a quality assurance, all spectra were visually inspected after LCModel fitting.

\section{Statistical analyses}

Since the investigated subjects were not considered to be normally distributed, a non-parametric approach was used in the statistical analyses. As previously described, the main focus of this report was on the cerebellar and thalamic GABA+ and Glx concentrations acquired from the left and right hemispheres. However, to increase the power of the measurements, a bilateral measurement, thus, an average of the left and right computed concentrations was also computed and investigated. Furthermore, the GABA+/Glx ratio was also investigated to exclude any potential issues that might result from the water scaling.

First, the unpaired non-parametric Mann-Whitney test was used to compare the computed concentrations and ratios obtained from the ET patient group to the resulting concentrations obtained from the healthy controls. Second, due to a previous reported cerebellar GABA+ asymmetry [23], the Mann-Whitney test was also used to investigate a potential thalamic or cerebellar asymmetry of GABA+ in the ET patient group. Third, correlation analyses were performed, correlating the GABA+ and Glx concentrations, and the GABA+/Glx ratio to the computed ETRS score defined previously. These correlation analyses were only performed for the ET patient group, and the Spearman correlation coefficients and corresponding $\mathrm{p}$-values were computed to show significance. Finally, a significance level of $\mathrm{p}=0.05$ was chosen for all analyses. No correction for multiple comparsions was applied.

\section{Results}

The ET patients and HCs were similar in demographic factors and in clinical variables such as ethanol intake and handedness. Table 1 shows the ETRS score for each patient, and Supplementary Table 1 summarizes the score from each ETRS item for each patient, evaluated for the patients' right upper extremity. The mean computed ETRS score over the ET patient group was $17.6 \pm 2.9$, which indicates a high overall tremor severity of this patient population. Meanwhile, all subjects in the healthy control group had an ETRS score of 0.

\section{GABA+ and Glx concentrations}

One of the patient's Glx-measurements (from the right thalamic voxel) was discarded after quality inspection of the fitted spectra. Therefore, the corresponding bilateral measurement was computed using only the left thalamic Glx concentration. All other spectra passed this visual inspection, and corresponding computed concentration were used in further analyses.

Figure 2 illustrates the average computed GABA+ and Glx concentrations, and also the GABA+/Glx ratios for the cerebellar (A) and thalamic (B) measurements. No significant differences between the ET and HC groups were observed in the bilateral, left and right GABA+ and Glx concentrations or in the corresponding GABA+/Glx ratios. Furthermore, a non-significant higher mean Glx concentration was observed in both the cerebellar and thalamic voxel placements for the HC group compared to the ET patients.

\section{Potential GABA+ asymmetry}

We did not observe any significant differences between GABA + concentrations in the left and right hemispheres (either for the thalamic $(\mathrm{p}=0.85)$ or the cerebellar $(\mathrm{p}=$ 0.35 ) voxels, Supplementary Figure 1). However, generally a higher right cerebellar GABA+ concentration was observed, which agrees with previously reported results [23]. We did not observe any lateral differences of thalamic GABA+ concentrations. 


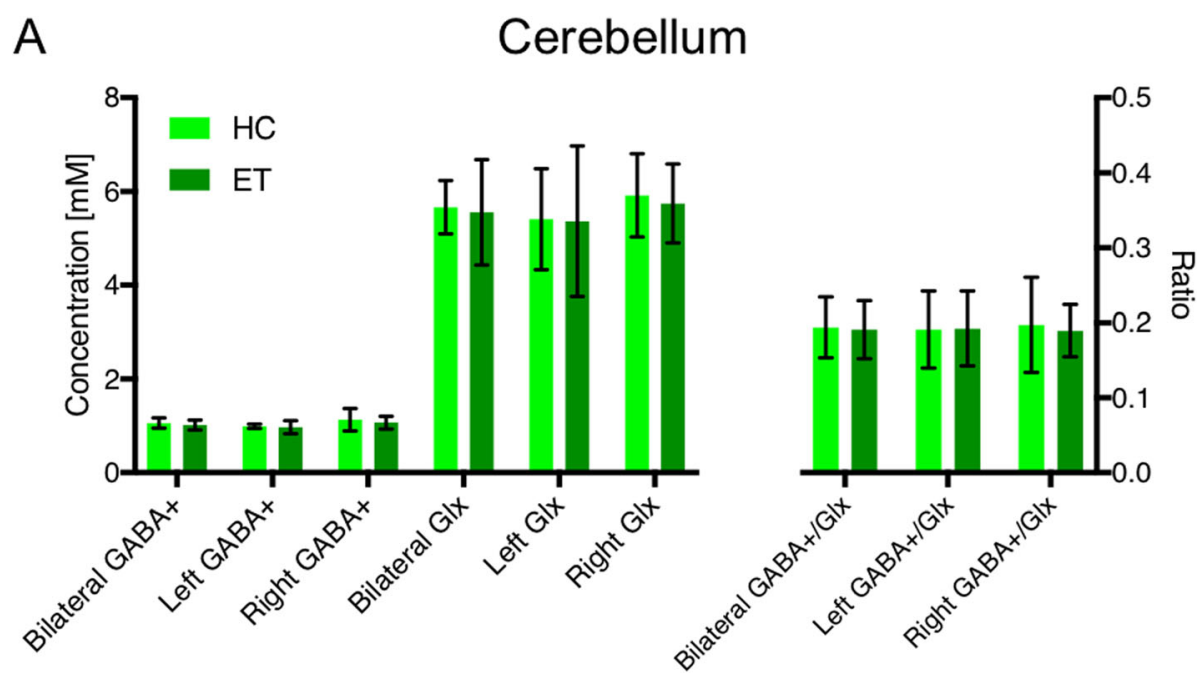

B

\section{Thalamus}

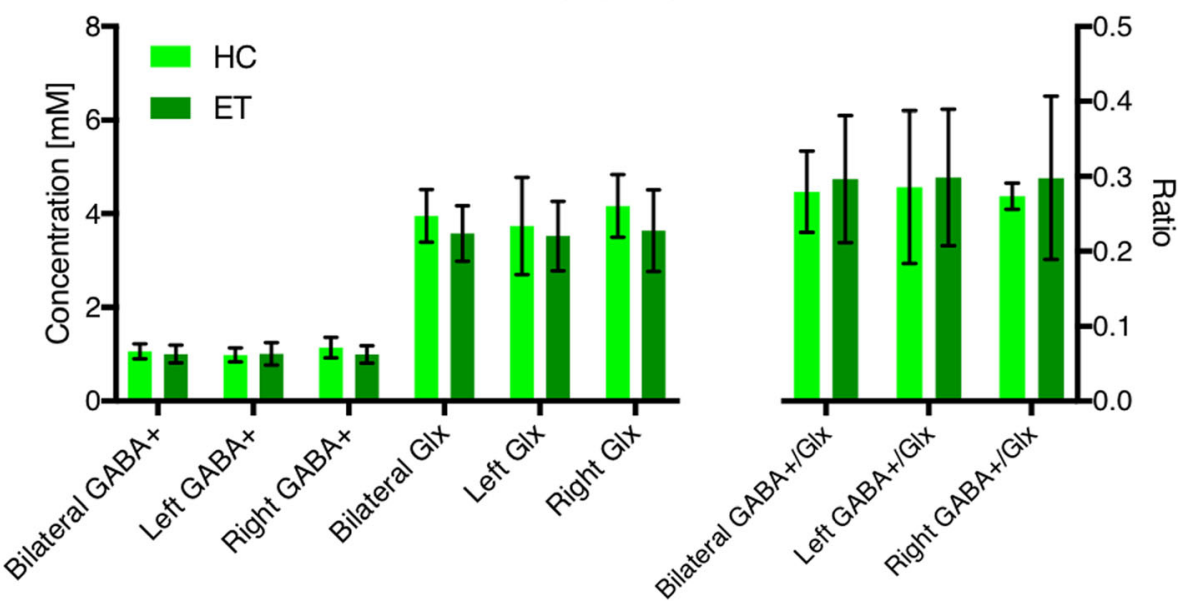

Fig. 2 MRS Concentrations. Cerebellar (a) and thalamic (b) GABA+ and Glx concentrations, and GABA+/Glx ratios reported as a mean $\pm S D$ over the measurements. The healthy control group (HC) is shown in light green, and the ET patient group (ET) is shown in dark green

Bilateral MRS concentrations correlated to ETRS score Bilateral cerebellar or thalamic GABA+ and Glx concentrations did not show any significant correlation to ETRS (Fig. 3, A-B and D-E). However, the cerebellar GABA+/Glx ratio was positively correlated to the ETRS ( $r=0.70, p=0.03$, Fig. 3c). This positive correlation was due to the reduction in cerebellar Glx and a small increase in cerebellar GABA+ with a higher tremor severity. No significant correlation was observed between thalamic GABA+/Glx ratios and tremor severity (Fig. 3f).

\section{Cerebellar GABA+/Glx correlated to ETRS score}

Since the bilateral GABA+/Glx ratio was positively correlated to the ETRS, the left and right sides are reported separately in Supplementary Figure 2. The right cerebellar GABA+/Glx ratio was significantly correlated to
ETRS $(\mathrm{r}=0.73, \mathrm{p}=0.02)$, whereas only a positive trend $(r=0.51, p=0.14)$ was observed for the left side.

\section{Discussion}

In the present study we have demonstrated the presence of neurometabolic changes in the cerebellum of patients with ET, which were correlated to ETRS. To our knowledge this is the first study to show a positive correlation between the ratio of GABA+ and Glx in the cerebellum of ET patients, and increasing tremor disability suggesting a pathological correlation. Our interpretation is that this can be attributed to an overall decrease of Glu, rather than an increase in GABA+, which was constant. This suggests that an increasing ETRS score is partly due to a disturbance in cerebellar Glu-concentration, and that there is a greater impact of low Glx concentration on tremor severity than a change of GABA+ 


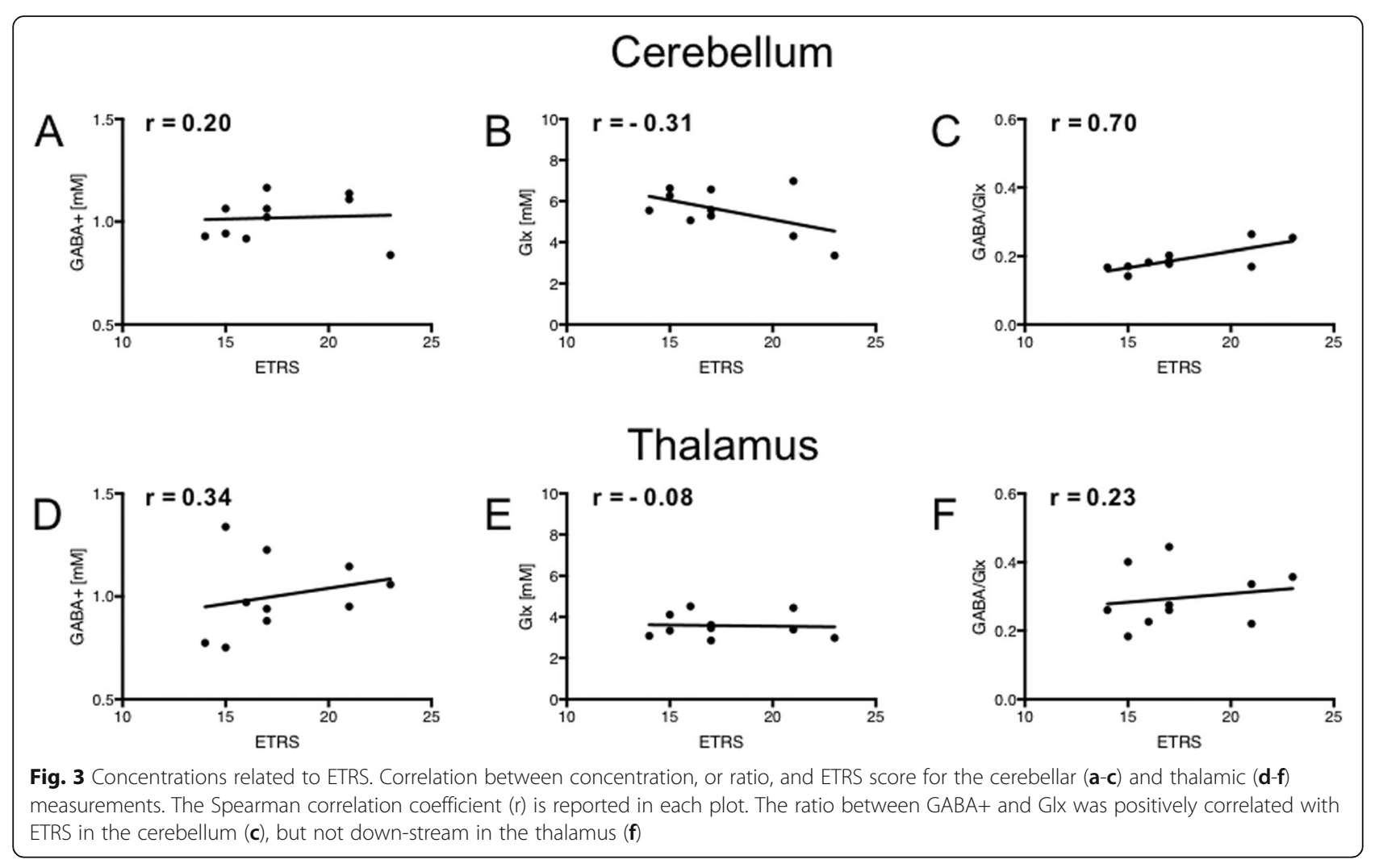

concentration. Moreover, a greater ratio between the two main neurotransmitters acting in an inhibitory manner $(\mathrm{GABA}+)$ and an excitatory manner (Glx) on the synaptic level respectively, seems to correlate with a more severe tremor. This highlights the importance of the balance between the two excitatory and inhibitory neurotransmitters for proper motor control.

\section{Spatial origin of disease}

Recent mechanistic research on ET has focused on anomalies localized in the cerebellum, in particular Purkinje cells and the dentate nucleus [33]; these sites are schematically labeled (1) and (2) in Fig. 4. Treatment of therapy resistant ET can include 'Deep Brain Stimulation' (DBS) of the ventral intermediate nucleus of the thalamus or the dentatorubrothalamic tract, and therefore both the cerebellum and thalamus were examined in this study. Moreover, the pathology in ET is not static, and the symptoms often progress and spread to other cerebellar areas [3]. In addition, previous reports have shown a correlation to increasing tremor severity with abnormalities; the latter included decreased cerebellar cortical NAA/tCr [34], CF-PC synaptic pathology [21], both suggesting cerebellar neurodegeneration. Also, increased thalamic Glx concentration and $\mathrm{Glx} / \mathrm{Cr}$ ratio values [35] have both been shown to correlate to tremor severity. Our findings suggest an additional, previously unreported, perspective on the pathological process in the cerebellum.

We did not find a significant difference in GABA concentration (see Fig. 2), which is in accordance with previous studies, in which a reduction of the number of GABA receptors was observed, but not a reduction in the cerebellar GABA concentration [17]. MEGA-PRESS MRS at $3 \mathrm{~T}$ in our application did not allow us to spatially separate signals from the dentate nucleus and cortical Purkinje cells, which is illustrated schematically by the red dotted square in Fig. 4. Thus, we have to interpret the results as a combination of the outcome of cellular events within all of these tissues (see Fig. 1).

\section{Aspects of tissue structure}

Tremor is the most prevalent movement disorder, but it represents a wide range of etiologies and it may represent a number of progressive diseases [36]. Moreover, clinical-, pathological and pharmacological heterogeneity may explain why evidence regarding etiology is difficult to conclude. A number of morphological aberrations have been identified in ET, mainly affecting Purkinje cells. In contrast, no pathological morphological changes have been found for other regions involved in the motor network (including the thalamus).

Previous observations include a reduced number of Purkinje cells (PCs), dendritic swelling and increased 


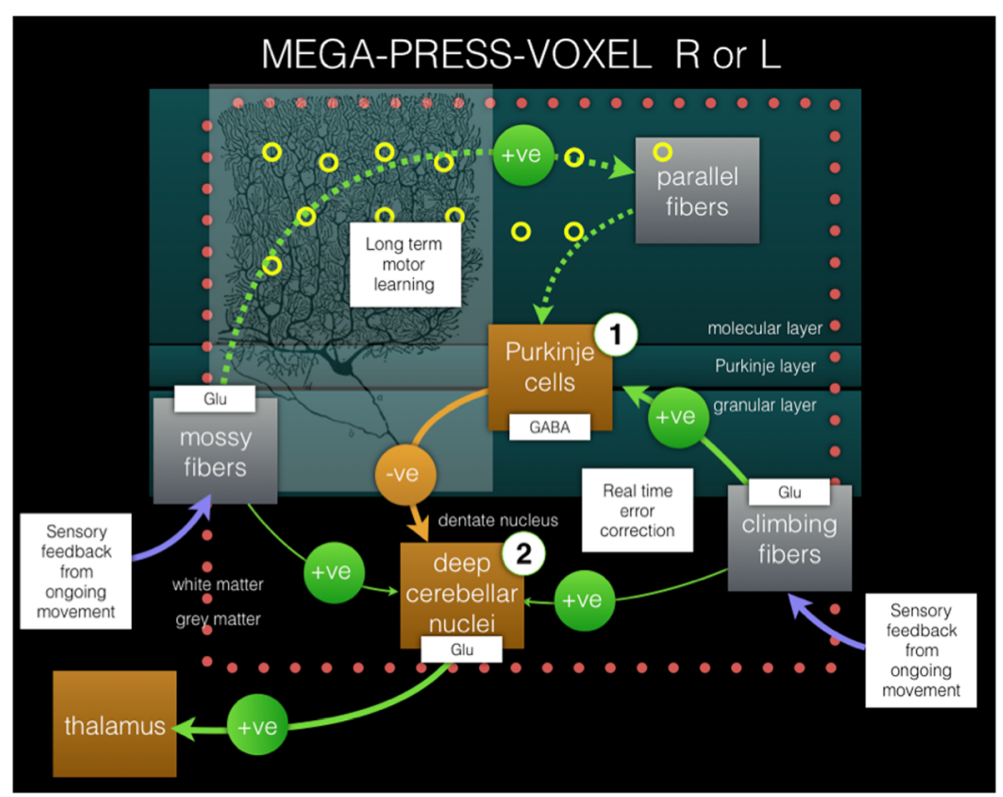

Fig. 4 Pathways in ET. Pathways of cerebellar error-correction in ET. The suggested neurodegeneration results in a relative decrease of excitatory neurotransmitter Glu, resulting in a hypothetical net decreased inhibitory effect from Purkinje cells on the dentate nucleus in ET. This will in turn lead to an imbalance in the cerebellar error correction, based on a relative decrease of the effect induced by the excitatory feedback response from the sensory system of ongoing movement. The MEGA-PRESS voxels were acquired at $3 T$, separately for the $R$ and $L$ cerebellum, including both the Purkinje layer, and the subcortical dentatus nucleus. The MEGA-PRESS-voxel (either R or L) is represented by a dotted square. Labels (1) and (2) represent two specifically reported locations of structural-functional aberrations in ET versus healthy controls (see Discussion for more details). Excitatory and inhibitory synaptic stimulation is represented by green and yellow circles, respectively. (The image of a Purkinje cell was obtained from Gray's anatomy, 1918 edition, in the public domain)

Purkinje cell torpedoes in the cerebellum [14], and an increased number of heterotopic Purkinje cells, localized in the molecular layer of the cerebellar cortex [37]. In contrast, a number of other studies have failed to show evidence of Purkinje cell loss [38, 39]. A difference in response to pharmacological substances further elucidates the heterogeneity of the disease.

\section{Is the disease symmetric, or not?}

Similar to our observations, Louis and co-workers [23] did not find a reduction in GABA+ concentration in the dentate nucleus of ET using MEGA-PRESS, compared to the control subjects. Asymmetries of metabolic changes in ET have previously been reported, and Louis and co-workers did find an asymmetry, as the concentration of GABA in the right dentate nucleus was c. $15 \%$ larger than the concentration on the left dentate nucleus. This also agrees with our observations as we did not detect a GABA reduction, but an asymmetric distribution of GABA in the cerebellar region of interest. The latter was, in the patient cohort described here, limited to a trend (representing c. $+10 \%$ concentration difference of GABA+, R vs. L), and therefore was not a significant effect.

In the context of symmetry, it is important to consider aspects such as objective asymmetry in clinical expression, asymmetry in perceived disability (cf. dominant hand), as well as tissue asymmetry in terms of tissue structure and biochemical parameters. Contralateral low thalamic NAA/Cr ratio was found in ET patients with predominantly tremor of the right arm [40], and clinical asymmetry of tremor was correlated to a significant degree with cerebellar pathology [41]. All of our patients perceived more symptomatic tremor on the right side, thus displaying an asymmetry from a clinical perspective. This may explain why significant correlation of GABA+/ Glx versus ETRS was only detected on the ipsilateral cerebellar side (see Supplementary Figure 2), although one potential limitation of this observation of the sideto-side difference may be the limited sample size.

\section{Inhibitory input}

The Purkinje cells convey their input to the deep cerebellar nuclei through GABA, the main inhibitory neurotransmitter, which binds to synaptic GABA receptors. Flumazenil, which specifically binds to the $\mathrm{GABA}_{\mathrm{A}}$ receptor, has been shown in PET studies to map the binding in the cerebellum (dentate nucleus), but also in the thalamus and the premotor cortex [18]. Other PET studies have also shown that the severity of tremor was correlated to abnormal GABA receptor binding [19]. In addition, a previous study showed a reduction of GABA 
receptors but not in GABA concentration in the cerebellum [17]. Available medication is very non-specific, but certain vectors that increase GABAergic transmission can be effective in treating ET, although with very variable outcomes [42].

Purkinje cells receive two direct excitatory inputs (Glu), from climbing fibers and from parallel fibers [43]. (See also Fig. 4) Notably, the synaptic distributions of the climbing fibers appear to be important to control Purkinje cell physiology and therefore normal cerebellar function. A particularly interesting finding, which affects the climbing fiber to Purkinje cell synaptic connections, was the observation that synaptic density in the molecular layer of the cerebellar cortex was associated with tremor severity [15]. A lower synaptic climbing fiber-Purkinje cell density would subsequently result in a lower degree of inhibition of the dentate nucleus. Furthermore, it has been shown that the excitatory amino acid transporter 2 (EAAT2) is reduced in the cerebellar cortex and increased in the dentate nucleus in patients with ET, indicating that the glutamatergic synaptic transmission is altered [44]. The combined post-mortem observations of these studies showed that the excitatory input (i.e., Glu-input) was disturbed both in the Purkinje cells and at the dentate nucleus in ET patients. Our interpretation is that the observed lower glutamate concentrations (expressed in terms of reduced Glx concentrations) further advocate the role of altered glutaminergic transmission in ET.

\section{What about the thalamus?}

Neurogenic excitatory output from the cerebellum is propagated to the thalamus, which is suggested to have a role in the pathogenesis of ET. Interestingly, the effects of a focal stroke can ameliorate the symptoms, as has been reported by Duncan [45]. Metabolic abnormalities with low NAA/Cr ratio of the contralateral thalamus in ET patients with predominantly tremor of the right arm have been reported [40]. In a recent study, the Glu-concentration was increased in the thalamus of ET patients and correlated to tremor severity, suggesting that thalamic glutamatergic transmission is involved in the pathophysiology [35]. No significant differences in thalamic GABA+, Glx or GABA+/Glx between the ET and HC groups were observed in our study. Furthermore no correlation with GABA+/Glx and tremor severity was seen in the thalamus. The voxel placed in our series is larger and extends beyond the thalamic anatomical borders, thus involving the brain areas in the vicinity of the thalamus (see Fig. 2). Our thalamic results are therefore not easily comparable with previous reports and this may be the reason why our results differ.

\section{Limitations}

Certain experimental aspects of this work were restricted by safety, hardware, partial volume and/or techniques. The patient cohort examined here were all diagnosed by a movement disorder specialist, and then referred to the neurosurgical department, thus they constituted a limited, but very narrow and well-defined group (bilateral, but right-handed tremor). Most of them had severely debilitating hand tremor and they were therefore all selected as being eligible for surgery. Due to the severely disabling disease, surgery was performed immediately after these measurements, with an excellent therapeutic outcome; all patients were also significantly better after surgery (data not shown), indicating the uniquely welldefined cohort.

Unfortunately, the measurements could not be repeated after the surgery, both for ethical and for safety reasons, as the acceptable exposure to electromagnetic fields of the DBS-implants was not compatible with the pre-surgical examination conditions (i.e., $3 \mathrm{~T}$ and the SAR level required), and this was a limitation of the present work. At a later time (likely within a few years?) when a sufficiently acceptable set of MR safety conditions have been developed, this may nevertheless be possible to perform.

When performing GABA detection, there are several technical aspects to be considered, one being the complex nature of the spectral scalar coupling pattern of the GABA resonances, and also due to the low signal intensity compared to the other metabolite signals in the spectra. Additionally, another major issue is spectral overlapping by the more intense creatine signal at 3.00 ppm, which obscures the GABA signal at $3.01 \mathrm{ppm}$ severely. In addition, spectral editing is needed to reveal the GABA+ signal, and single voxel MEGA-editing is currently the standard technique. Moreover, as we are quantifying difference spectra (using water scaling in LCModel), a high signal-to-noise ratio (SNR) is necessary; thus, we need a sufficiently large number of signal averages and also a large spectral voxel to achieve a high enough SNR for reliable concentration quantification of the resulting spectra. In comparison with conventional MRS, MEGA-PRESS requires a substantially larger voxel volume, which results in tissue heterogeneity between Purkinje cells and the dentate nucleus etc.

Compared to Louis et al. [23] we used a larger voxel in our cerebellar measurements $\left(35 \times 25 \times 25 \mathrm{~mm}^{3}\right.$, which is equivalent to $21.9 \mathrm{~mL}$ compared to $25 \times 25 \times 25 \mathrm{~mm}^{3}$, $15.6 \mathrm{~mL}$ ). Thus, in our measurements of cerebellar tissue we analyzed part of the cerebellar cortex, in combination with the deep cerebellar nuclei and white matter tracts in the cerebellum. Furthermore, to compensate for the large SNR reduction when using a smaller voxel size, Louis et al. collected more signal averages (196 ON/OFF 
compared to our 160 ON/OFF). In addition, there might also be other systematic differences between our results since we used different MR systems (Philips compared to Siemens), and different LCModel basis sets were used in the quantification. Our experience is that there are small, but noticeable, systematic differences in spectral analysis when different basis sets are used. Furthermore, Louis et al. used segmentation to determine the tissue composition of their voxel, a procedure which we did not apply. However, our interpretation is that most of these effects are removed when concentration ratios are used, and thus we found a significant correlation between cerebellar GABA+/Glx and ETRS. Although these differences in acquisition and analytical approach exist, the results should in our view still be comparable.

Our present data does not let us to come to a conclusion on whether ET is characterized by such morphological abnormalities in specific cerebellar regions, or whether the biochemical alterations are limited to certain parts, or widespread, as the procedures used here were limited with respect to the spatial resolution of the MRS-technique utilized. However, with increasing advances in MRS technology the voxel volume can be reduced, allowing different regions to be separately analyzed. One such procedure is 3D spiralMEGA-PRESS GABA Chemical Shift Imaging devised by Bogner et al. [46], for whole brain detection of GABA + at a spatial resolution of about $1 \mathrm{~cm}$ (compared to $3 \mathrm{~cm}$ which was used here); the technique requires a uniquely stable spiral acquisition. Another procedure is the Glu-CEST procedure championed by Reddy et al. for detecting Glu-weighted images of the excitatory neurotransmitter with the spatial resolution of conventional imaging [47]. However, both techniques are extremely demanding on subjects, hardware (Glu-CEST requires $7 \mathrm{~T}$ ) as well as operator skills, and at this time they are only available to selected laboratories.

Since we base our argument on the ratio between GABA+ and Glx, it is of interest to consider what we have measured. The GABA+ measurements include a proportion of macromolecules, and probably also homocarnosine, which is often considered to be a constant fraction (about 40-50\%); thus, any short-term changes in GABA+ mainly reflect changes in GABA. Glx is also a composite but of Glu and Gln, although a majority of the signal typically represents Glu [48]. The largest Glu to Gln ratios at the cellular level have been observed in excitatory terminals of parallel fibers in the molecular layer, and also in mossy and climbing fibers, corresponding to a concentration ratio of about $80 \%$ Glu (of Glx); and the lowest ratios have been observed in glial cells [49]. Another parameter to consider is that most Gln in the brain is less metabolically active than Glu. Please also note that MRS is typically acquired at a much lower spatial resolution, and the partial volume effects of a wide range of different cell populations then make it harder to predict a neurotransmitter ratio accurately based on cellular concentrations. However, our interpretation is that the majority of the Glx-resonances in the cerebellum are indeed Glu, and this agrees with a report by Goryawala which determined a concentration ratio of between 2 and 4 in gray matter, corresponding to c. $70 \%$ Glu (of Glx) in the cerebellum and close $80 \%$ in the brain [50].

Determination of absolute concentrations is typically recommended as this will facilitate comparison between different tissues and subjects [22]. Usually, this is achieved by the use of LCModel analysis in combination with water scaling, which is much preferred to creatine-ratios. In the cerebellum, in consideration of the relative tissue heterogeneity, water scaling appears to be less effective due to the spatial (including intra vs. extra-cellular) tissue variation of water content. This is the likely cause of the relatively large variation of the GABA+ and Glx concentrations in Fig. $3 \mathrm{a}$ and $\mathrm{b}$. Instead, the uniquely functionally coupled ratio between the related neurotransmitters GABA and Glu will remove the effects of such spatial variation (see Fig. 3c). It is also worth noting that another difference between our study and some others, was the limited number of included ET patients and healthy control subjects (here $10 \mathrm{ET}$ patients and six $\mathrm{HCs}$ ). However, our patients belonged to a highly selective cohort of ET patients intended for DBS therapy, which may show stronger disease-related parameters.

\section{Conclusions}

This is to our knowledge the first study to show a correlation between the GABA+/Glx ratio in the cerebellum and increasing tremor disability. A change of balance between excitatory and inhibitory neurotransmitters related to a tremor rating scale (ETRS) could explain part of the pathophysiology of ET. This is rather attributed to a decrease in tissue Gluconcentration, than an altered GABA+-concentration, suggesting that an increasing ETRS score is partly due to a disturbance in cerebellar Glu concentration. Importantly, we interpret this as being a consequence of an overall decreased stimulatory effect on the inhibitory Purkinje cells, subsequently leading to decreased influence on nucleus dentatus driving the tremor. This finding may open up a range of entirely new therapeutic alternatives, which so far have been largely unexplored. The exact neurochemical abnormalities underlying ET remain to be elucidated in a larger study. 


\section{Supplementary information}

Supplementary information accompanies this paper at https://doi.org/10. 1186/s40673-020-00116-y.

\section{Additional file 1. \\ Additional file 2.}

Additional file 3

\section{Acknowledgements}

Richard Edden is gratefully acknowledged for very generously providing the MEGA-PRESS pulse sequence and other tools that were developed under NIH GRANTS P41 015909 and R01 016089.

\section{Authors' contributions}

PZ, PL, AT, NG and ST contributed to the conception and design of the study. PZ, NG and AT were responsible for recruting the patients and healthy controls and for the clinical interviews. PZ, AT, NG and ST were responsible for data acqusition and statistical analysis. All authors were responsible fore preparing the manuscript and all authors approved the final version of the article.

\section{Funding}

This research was funded by the County of Östergötland. The research did not receive grant from the commercial sector. Open access funding provided by Linköping University.

\section{Availability of data and materials}

The MRI and clinical data that support the findings in this study are available on reasonable request from ST, AT and the last author PZ. Raw data are not publicly available due to regulations of the County of Östergötland.

\section{Ethics approval and consent to participate}

The study was approved by the by the local ethics committee at the University Hospital in Linköping (Ref. No. 2013/403-31, P. Zsigmond), and informed written consent was received from the patients and healthy controls.

\section{Competing interests}

The authors of this article declare no competing interests.

\section{Author details}

${ }^{1}$ Center for Medical Image Science and Visualization (CMIV), Linköping University, Linköping, Sweden. ${ }^{2}$ Department of Medical Radiation Physics and Department of Health, Medicine and Caring Sciences, Linköping University, Linköping, Sweden. ${ }^{3}$ Department of Biomedical Engineering, Linköping University, Linköping, Sweden. ${ }^{4}$ Department of Neurosurgery and Department of Biomedical and Clinical Sciences, Linköping University, Linköping, Sweden.

\section{Received: 3 April 2020 Accepted: 29 May 2020}

Published online: 26 June 2020

\section{References}

1. Louis ED, Ferreira JJ. How common is the most common adult movement disorder? Update on the worldwide prevalence of essential tremor. Mov Disord. 2010;25(5):534-41.

2. Klaming $R$, Annese J. Functional anatomy of essential tremor: lessons from neuroimaging. AJNR Am J Neuroradiol. 2014;35(8):1450-7.

3. Rajput A, Robinson CA, Rajput AH. Essential tremor course and disability: a clinicopathologic study of 20 cases. Neurology. 2004;62(6):932-6.

4. Louis ED. Essential tremors: a family of neurodegenerative disorders? Arch Neurol. 2009:66(10):1202-8.

5. Sharifi $\mathrm{S}$, et al. Neuroimaging essentials in essential tremor: a systematic review. Neuroimage Clin. 2014;5:217-31.

6. Benito-León J, Louis ED, Romero JP, et al. Altered functional connectivity in essential tremor: a resting-state fMRI study. Medicine (Baltimore). 2015; 94(49):e1936.

7. Lenka $A$, et al. Role of altered cerebello-thalamo-cortical network in the neurobiology of essential tremor. Neuroradiology. 2017;59(2):157-68.
8. $\quad \mathrm{Cao} \mathrm{H}_{\text {, et }}$ al. A voxel-based magnetic resonance imaging morphometric study of cerebral and cerebellar gray matter in patients under 65 years with essential tremor. Med Sci Monit. 2018;24:3127-35.

9. Contarino MF, et al. Is there a role for combined EMG-fMRI in exploring the pathophysiology of essential tremor and improving functional neurosurgery? PLoS One. 2012;7(10):e46234.

10. Jenkins $\mathbb{H}$, et al. A positron emission tomography study of essential tremor: evidence for overactivity of cerebellar connections. Ann Neurol. 1993;34(1): $82-90$

11. Singer C, Sanchez-Ramos J, Weiner WJ. Gait abnormality in essential tremor. Mov Disord. 1994:9(2):193-6.

12. Deuschl G, Wenzelburger R, Raethjen J. Tremor. Curr Opin Neurol. 2000; 13(4):437-43.

13. Critchley E. Clinical manifestations of essential tremor. J Neurol Neurosurg Psychiatry. 1972;35(3):365-72.

14. Louis ED, et al. Neuropathological changes in essential tremor: 33 cases compared with 21 controls. Brain. 2007;130(Pt 12):3297-307.

15. Lin CY, et al. Abnormal climbing fibre-Purkinje cell synaptic connections in the essential tremor cerebellum. Brain. 2014;137(Pt 12):3149-59.

16. Lee $D$, et al. Climbing fiber-Purkinje cell synaptic pathology across essential tremor subtypes. Parkinsonism Relat Disord. 2018;51:24-9.

17. Paris-Robidas $S$, et al. Defective dentate nucleus GABA receptors in essential tremor. Brain. 2012;135(Pt 1):105-16.

18. Boecker $\mathrm{H}$, et al. GABAergic dysfunction in essential tremor: an 11Cflumazenil PET study. J Nucl Med. 2010;51(7):1030-5.

19. Gironell A, et al. Gaba and serotonin molecular neuroimaging in essential tremor: a clinical correlation study. Parkinsonism Relat Disord. 2012;18(7):876-80.

20. Marin-Lahoz J, Gironell A. Linking essential tremor to the cerebellum: neurochemical evidence. Cerebellum. 2016;15(3):243-52.

21. Louis RJ, et al. Climbing fiber synaptic changes correlate with clinical features in essential tremor. Neurology. 2015;84(22):2284-6.

22. Tisell $\mathrm{A}$, et al. Procedure for quantitative (1) $\mathrm{H}$ magnetic resonance spectroscopy and tissue characterization of human brain tissue based on the use of quantitative magnetic resonance imaging. Magn Reson Med. 2013;70(4):905-15.

23. Louis ED, et al. In vivo dentate nucleus gamma-aminobutyric acid concentration in essential tremor vs. Controls. Cerebellum. 2018;17(2):16572.

24. Fahn S, TE, Marin C. Clinical rating scale for tremor. Munich: Urban and Schwarzenberg; 1993.

25. Mullins PG, et al. Current practice in the use of MEGA-PRESS spectroscopy for the detection of GABA. Neuroimage. 2014:86:43-52.

26. Mescher $\mathrm{M}$, et al. Simultaneous in vivo spectral editing and water suppression. NMR Biomed. 1998;11(6):266-72.

27. Mescher $M$, et al. Solvent suppression using selective Echo Dephasing. J Magn Reson Ser A. 1996;123(2):226-9.

28. Tapper S, Tisell A, Lundberg P. How does motion affect GABAmeasurements? Order statistic filtering compared to conventional analysis of MEGA-PRESS MRS. PLoS One. 2017:12(5):e0177795.

29. Klose U. In vivo proton spectroscopy in presence of eddy currents. Magn Reson Med. 1990:14(1):26-30.

30. Provencher SW. Estimation of metabolite concentrations from localized in vivo proton NMR spectra. Magn Reson Med. 1993:30:672-9.

31. Dydak $U$, et al. In vivo measurement of brain GABA concentrations by magnetic resonance spectroscopy in smelters occupationally exposed to manganese. Environ Health Perspect. 2011;119(2):219-24.

32. Govindaraju V, Young K, Maudsley AA. Proton NMR chemical shifts and coupling constants for brain metabolites. NMR Biomed. 2000;13(3):129-53.

33. Passamonti L, Cerasa A, Quattrone A. Neuroimaging of Essential Tremor: What is the Evidence for Cerebellar Involvement? NY: Tremor Other Hyperkinet Mov; 2012. p. 2

34. Louis ED, et al. Metabolic abnormality in the cerebellum in patients with essential tremor: a proton magnetic resonance spectroscopic imaging study. Neurosci Lett. 2002:333(1):17-20.

35. Barbagallo $\mathrm{G}$, et al. Increased glutamate + glutamine levels in the thalamus of patients with essential tremor: a preliminary proton MR spectroscopic study. Parkinsonism Relat Disord. 2018;47:57-63.

36. Louis ED. Understanding essential tremor: progress on the biological front. Curr Neurol Neurosci Rep. 2014;14(6):450.

37. Kuo SH, et al. Increased number of heterotopic Purkinje cells in essentia tremor. J Neurol Neurosurg Psychiatry. 2011;82(9):1038-40. 
38. Rajput AH, Rajput A. Significance of cerebellar Purkinje cell loss to pathogenesis of essential tremor. Parkinsonism Relat Disord. 2011;17(6):410-2.

39. Symanski C, et al. Essential tremor is not associated with cerebellar Purkinje cell loss. Mov Disord. 2014;29(4):496-500.

40. Kendi AT, et al. Magnetic resonance spectroscopy of the thalamus in essential tremor patients. J Neuroimaging. 2005;15(4):362-6.

41. Louis ED, et al. Matching asymmetry of tremor with asymmetry of postmortem cerebellar hemispheric changes in essential tremor. Cerebellum. 2014;13(4):462-70.

42. Bruno E, et al. Pregabalin for essential tremor. Cochrane Database Syst Rev. 2016;10:Cd009682

43. Hirano T. Purkinje neurons: development, morphology, and function. Cerebellum. 2018;17(6):699-700.

44. Wang J, et al. Excitatory amino acid transporter expression in the essential tremor dentate nucleus and cerebellar cortex: a postmortem study. Parkinsonism Relat Disord. 2016;32:87-93.

45. Duncan R, Bone I, Melville ID. Essential tremor cured by infarction adjacent to the thalamus. J Neurol Neurosurg Psychiatry. 1988;51(4):591-2.

46. Bogner W, et al. 3D GABA imaging with real-time motion correction, shim update and reacquisition of adiabatic spiral MRSI. Neuroimage. 2014;103: 290-302.

47. Bagga P, et al. In vivo GluCEST MRI: reproducibility, background contribution and source of glutamate changes in the MPTP model of Parkinson's disease. Sci Rep. 2018;8(1):2883.

48. Rae CD. A guide to the metabolic pathways and function of metabolites observed in human brain $1 \mathrm{H}$ magnetic resonance spectra. Neurochem Res. 2014;39(1):1-36

49. Ottersen OP, Zhang N, Walberg F. Metabolic compartmentation of glutamate and glutamine: morphological evidence obtained by quantitative immunocytochemistry in rat cerebellum. Neuroscience. 1992;46(3):519-34.

50. Goryawala MZ, Sheriff S, Maudsley AA. Regional distributions of brain glutamate and glutamine in normal subjects. NMR Biomed. 2016;29(8): 1108-16.

\section{Publisher's Note}

Springer Nature remains neutral with regard to jurisdictional claims in published maps and institutional affiliations.

Ready to submit your research? Choose BMC and benefit from:

- fast, convenient online submission

- thorough peer review by experienced researchers in your field

- rapid publication on acceptance

- support for research data, including large and complex data types

- gold Open Access which fosters wider collaboration and increased citations

- maximum visibility for your research: over $100 \mathrm{M}$ website views per year

At $\mathrm{BMC}$, research is always in progress.

Learn more biomedcentral.com/submissions 